\title{
On the proximity relation between two surface-melted clusters involved in inter-cluster mass-transfer
}

\author{
F. Despa and R.S. Berry \\ Department of Chemistry, \\ The University of Chicago, \\ Chicago, Illinois 60637
}

October 27, 2000

\begin{abstract}
We explore the way free particles produced by dissociating "particlehole pairs" on a surface-melted cluster can be transferred to a second, nearby surface-melted cluster. This mass transport is based on an inter-cluster direct transfer mechanism of the particles. We found that in this particular case one cluster may grow at the expense of another, obeying a temporal power law with the exponent $1 / 2$ for the average radius $\left(R \sim t^{1 / 2}\right)$. The change from the expected universal power law $\left(R \sim t^{1 / 3}\right)$ is a consequence of the proximity relation between these two clusters which lead to enhance the effective transport rates.
\end{abstract}

It is widely agreed that, under certain conditions, clusters may exhibit more than two phaselike forms coexisting in dynamic equilibrium. [1, 2, 2, 3, 国, 国] For example, $A r_{55}$ is expected to show, depending on the temperature range, coexistence of solidlike, homogeneously melted forms and surface-melted either in an ensemble at any instant or in the time history of a single cluster. 圆, 5] It is also equally possible, e.g., for the solid form to be in equilibrium with only the surface-melted form and for the surface-melted form 
to coexist at higher temperatures with the liquid. There may or may not be a temperature range in which the surface-melted form is the only stable species.

The surface-melting phenomenon is usually exhibited in bulk matter and in large clusters (with about 50 or more particles, usually) at temperatures or energies a little below the temperatures or energies of homogeneous melting. This process involves local minima on the potential energy surface of the system corresponding to one or a few of the particles from the surface layer moving into local sites above the surface. The promoted particles are rather free and float on the cluster surface while all the other particles in the surface layer undergo large-amplitude, anharmonic oscillations. The numerical experiments indicate that the surface is liquidlike and all the particles of the surface, including the floaters, do permute among themselves without involving inner layers of the cluster.

The statistical mechanical underpinning of these findings allow for one other kind of behavior yet to be observed. This would be a dynamical process within which free particles produced by dissociating "particle-hole pairs" on a surface-melted cluster are allowed to recombine with available vacancies on a second, nearby surface-melted cluster. The inter-cluster direct mass transfer arisen in this way involves pairs of surface-melted clusters in a dense cluster ensemble. The occurrence of this process is limited by the energetic need to drive the particle flow and by an obvious requirement on the nearest-neighbor distance. We assume the surface-melted clusters are close to each other such that the direct transfer of the particles makes sense. The escape energy for the itinerant particle is achieved by thermal excitation and possible interaction with the surface vacancies on the neighbor cluster. The probability of transfer is high if the solid angle in the direction of jump is large. Therefore, the net flow of particles proceeds from the small cluster, with large curvature, to the large cluster in the pair, which has smaller curvature. Note that, an increase of the nearest-neighbor distance between surface-melted clusters over a critical value may lead to change the transport mechanism and sets in crossover phenomena.

The investigation of the direct mass transfer between neighboring surfacemelted clusters is of both theoretical and experimental interest. First of all, this is a useful way to increase our understanding of phase separation, coarsening [6] and crossover phenomena. [7, 8, 9, 10] The process can also have some relevance in describing kinetic transformations of the cluster- 
assembled materials. Second, the direct transfer mechanism of particles between surface-melted clusters offers the prototype of grain growth which deviates from the expected universal power law $\left(S \sim t^{2 / 3}\right)$ of the classical Ostwald ripening theory. [6] Generalization for solid clusters is straightforward.

Making reference only to the cluster-pair problem appears as a strong limitation in the broad context as declared above. Usually, more than two individuals in the entire cluster ensemble may act in this process. We believe that, for a dense cluster distribution, the chief particle transfer occurs, anyway, between nearest neighboring clusters, along the shortest separation distance where the particle concentration gradient is greatest possible.

It is the purpose of this work to explore the dynamics of such a process. The modelling starts by assuming that the separation distance between the surface-melted clusters involved in this process, say $\xi$, is of order of magnitude of the characteristic diffusion length $l(\xi \simeq l)$. In this context, the particles released from one cluster may reach the second, nearby cluster by performing an inter-cluster direct jump. Next, we assume that the surfacemelting process produces an amount of "particle-hole pairs" at the surface of each cluster. The number $M$ of "particle-hole pairs" is in direct proportion with the number of atoms on the cluster surface of area $S$ and depends on the temperature $T$

$$
M \simeq S \sigma \exp \left(-\frac{E_{p-h}}{k_{B} T}\right),
$$

where $\sigma$ stands for the surface particle density, $E_{p-h}$ is the energy to excite a particle above the melted surface (the "particle-hole pair" creation energy) and $k_{B}$ has the usual meaning. The floating particles perform continuous permutations (atomic scale jumps) among energetically equivalent sites (vacancies) on the cluster surface with the frequency

$$
v_{0}=v_{p-h} \exp \left(-\frac{E_{0}}{k_{B} T}\right),
$$

where $v_{p-h}$ is the vibration frequency of the "particle-hole pair" and $E_{0}$ is the energy cost of the "on-site" jump. The equivalent sites on the surface of this cluster are labelled by $i, i=\overline{1, M}$.

According to numerical experiments, 近, [0] the floating particles are rather free and get easily dissociate from the cluster surface. We suppose these particles can jump to the nearest neighbor cluster, over the separation distance 
$\xi$, where may recombine with vacancies of the host melted surface. The equivalent sites on the surface of the second, nearby, cluster (of surface area $S^{*}$ ) are labelled by $k, k=\overline{1, M^{*}}$ with $M^{*}$ given by

$$
M^{*} \simeq S^{*} \sigma \exp \left(-\frac{E_{p-h}}{k_{B} T}\right) .
$$

The "inter-site" transfer frequency is denoted by

$$
v \simeq v_{p-h} \exp \left(-\frac{E_{d}}{k_{B} T}\right),
$$

where $E_{d}$ stands for the energy cost to dissociate the "particle-hole pair".

We place now one cluster at $x$ and the other at $x+\xi$ and apply to this mass-transfer process a kinetic approach based on a system of master equations which describe both permutations and escapes of itinerant particles. 111 Accordingly, the rate of change of the particle number at every site "i" on the melted surface of the cluster placed at $x$ may be written as

$$
\begin{aligned}
\frac{\partial}{\partial t} n_{i}(x, t)= & v_{0} \sum_{i \neq j=1}^{M} n_{j}(x, t)-v_{0} \sum_{i \neq j=1}^{M} n_{i}(x, t)+ \\
& v \sum_{k=1}^{M^{* \prime}} n_{k}(x+\xi, t)-v \sum_{k=1}^{M^{* \prime}} n_{i}(x, t) \quad .
\end{aligned}
$$

After some simplifications, this becomes

$$
\begin{aligned}
\frac{\partial}{\partial t} n_{i}(x, t)= & v_{0} \sum_{i \neq j=1}^{M}\left[n_{j}(x, t)-n_{i}(x, t)\right]+ \\
& v \sum_{k=1}^{M^{* \prime}} n_{k}(x+\xi, t)-v M^{* \prime} n_{i}(x, t) .
\end{aligned}
$$

The first term on the right hand side of the equation accounts for the movement of the particles on the surface of the cluster (the number of floaters is conserved by this movement) while, the second term includes all transfer possibilities of the particles to the second, nearby cluster (naturally, this transfer changes the particle number at each site $i$ ). Obviously, those sites on cluster surfaces which do not face each other are excepted from the calculus. Therefore, $M^{* \prime}$ counts the effective available sites on the surface of the cluster 
placed at $x+\xi$ which can be reached by a direct jump, $M^{* \prime}=f M^{*}$ where $f$ is the weight of the geometrical obstruction (roughly, $f \simeq \frac{1}{2}$ ). Itinerant particles, subject of "inter-site" jumps, distribute themselves, quickly, over the whole surface area by "on-site" jumps (with the frequency $v_{0}, v_{0} \gg v$ ). In this way, all the "particle-hole pairs" get involved in the transport process. By summing up over all sites " $i$ " in (6) we obtain

$$
\frac{\partial n}{\partial t}=v_{b} n^{*}-v_{f} n,
$$

which is the transport equation for the particle number $n(x, t)=\sum_{i=1}^{M^{\prime}} n_{i}(x, t)$ with $n^{*}(x+\xi, t)=\sum_{k=1}^{M^{* \prime}} n_{k}(x+\xi, t)$. $v_{f}$ and $v_{b}$ stand for the forward and backward effective transition rates

$$
\begin{aligned}
& v_{f} \simeq \frac{1}{2} M^{*} v=\frac{S^{*} \sigma}{2} v_{p-h} e^{-\frac{E}{k_{B} T}} \\
& v_{b} \simeq \frac{1}{2} M v=\frac{S \sigma}{2} v_{p-h} e^{-\frac{E}{k_{B} T}},
\end{aligned}
$$

where $E=E_{d}+E_{p-h}$. The same set of equations $(5-7)$ apply for the cluster placed at $x+\xi$ and one get straightforwardly the transport equation for the particle number $n^{*}(x+\xi, t)=\sum_{k=1}^{M^{* \prime}} n_{k}(x+\xi, t)$ as

$$
\frac{\partial n^{*}}{\partial t}=v_{f} n-v_{b} n^{*} .
$$

By looking at equations $(7-9)$ we may infer that the net flow of itinerant particles occurs in the direction of the large cluster where the solid angle of the particle jump is large. For example, we may consider that at the initial moment of time $t=0$, the cluster placed at $x+\xi$ is larger by comparing with the other, $S^{*}>S$, which means that $v_{f}>v_{b}$. Consequently, the cluster placed at $x+\xi$ starts growing on the expense of the other which shrinks in time. The corresponding variation in time of the cluster surfaces $S^{*}$ and $S$ have to be in direct proportion with the instant numbers of particle concentrations $n$ and $n^{*}$ given by kinetic equations $(7-9)$ and should depend on the rate of spreading over the cluster surface (this is given by the transfer frequency $v_{0}$, subject of eq. 2). The fact can be expressed by the following set of coupled differential equations

$$
\begin{aligned}
& \frac{1}{A} \frac{d S^{*}}{d t}=n^{*}(t) ; \frac{1}{A} \frac{d S}{d t}=-n(t) \\
& \frac{1}{B} \frac{\partial n^{*}}{\partial t}=n S^{*}-n^{*} S ; \frac{1}{B} \frac{\partial n}{\partial t}=n^{*} S-n S^{*},
\end{aligned}
$$


where the forward and backward transition rates $v_{f, b}$ in above were replaced by (8) and use has been made of eq. (2) which render for the constants $A$ and $B$

$$
A=\frac{v_{p-h}}{\sigma} e^{-\frac{E_{0}}{k_{B} T}} ; \quad B \simeq \frac{\sigma v_{p-h}}{2} e^{-\frac{E}{k_{B} T}} .
$$

The evolution in time of cluster sizes depends on the degree of melting of their surfaces by the corresponding energy cost $E=E_{p-h}+E_{d}$ and by the characteristic (anharmonic) oscillation frequency $v_{p-h}$. In Fig. 1, we displayed the evolution in time of the surface area for the growing cluster, $S^{*}(t)$, following from the inter-cluster direct transfer mechanism of particles with the growth law given by eqs. (10) (the curve $a$ ). We may roughly approximate the growth law goes linearly $\left(S^{*} \sim t\right)$, which means the increase of the average radius of the cluster obeys a power law with the exponent equal to $1 / 2$. 12

We may observe that, actually, the set of eqs. (10) are general equations and can be used to describe the effective mass-transfer in any situation involving pairs of clusters which are not necessarily surface-melted but satisfy the appropriate proximity requirement $(\xi \simeq l)$. In the general case, $E$ is simply the dissociation energy for an atom at the cluster surface and $v_{p-h}$ becomes the surface vibration frequency $(v)$.

The result obtained above refers to the mechanism of direct transfer of particles between neighboring clusters. In principle, the process can switch to an asymptotic transport regime by increasing the separation distance between the two clusters. For widely separated clusters, the latter case resumes to an ideal one, where there is only one cluster growing directly from solution. The cluster growth obeys, in this case, the well-known power law

$$
R \sim t^{1 / 3},
$$

for the average radius and is known as the Ostwald ripening process. [6]] The growth of surface area goes as $\sim t^{2 / 3}$, in this case. For comparison, we displayed in Fig. 1 the growth law obeying the 2/3 power law (see the curve b).

The asymptotic regime occurs naturally in our model by letting the separation distance $\xi$ between clusters go to infinity $(\xi \gg l)$ which allows the itinerant particles to proceed by a random walk. By looking above we can see that this crossover between the direct particle transfer regime and the asymptotic one can be set up by transforming the kinetic equation (7) into 
a diffusion-like type of equation. This transformation requires $v_{f}=v_{b} \equiv v$ and a Taylor expansion up to second order of the concentration functions. The procedure allows to obtain the diffusion coefficient $\left(D \sim v l^{2}\right)$ which is the constant associated with the diffusion through the background matrix at large distances from the shrinking cluster. The rate of growth is then given by (12). Of course, the transition to the asymptotic regime proceeds gradually, being characterized by various transient values of the time exponents in the range $1 / 2$ through $1 / 3$. Finally, we can note that the main factor of delaying the setting up of the asymptotic regime is the proximity relation between the two clusters involved in the mass-transfer.

As one can see, the limiting regime $\left(R \sim t^{1 / 2}\right)$ identified here in the cluster growth process, namely the inter-cluster direct transfer of particles, has no relevant statistical aspects: this is a particle transfer process between two neighboring surface-melted clusters (therefore, essentially a cluster-pair problem). The statistical size distribution of clusters may change only irrelevantly at its two ends, where the small and the large clusters are placed. The statistical aspects are significant, in the sense that the distribution is much and fastly affected, in the transient regimes intervening between the two limiting ones $\left(R \sim t^{1 / 2}, R \sim t^{1 / 3}\right)$ described above.

\section{References}

[1] V.V. Nauchitel and A.J. Pertsin, Mol. Phys. 401341 (1980)

[2] H.-P. Cheng and R.S. Berry, in Proceedings of the Symposium on Clusters and Cluster-Assembled Materials, 1991, R. Averbach, Ed. (Materials Research Society, Pittsburgh, 1991), p. 241

[3] H.-P. Cheng and R.S. Berry, Phys. Rev. A 457969 (1992)

[4] R.E. Kunz and R.S. Berry, Phys. Rev. Lett. 713987 (1993)

[5] R.E. Kunz and R.S. Berry, Phys. Rev. E 491985 (1994)

[6] See, for example, S. W. Koch, Dynamics of First-Order Phase Transitions in Equilibrium and Non-Equilibrium Systems, in Lecture Notes in Physics, 207, Springer, Berlin (1984); P.W. Voorhees, Annu. Rev. 
Matter Sci. 22, 197 (1992) and A.J. Bray, Adv. Phys. 43357 (1994) for review of the traditional Lifshitz-Slyozov-wagner theory. Other new approaches employing the renormalization - group method are studied in P.C. Hohenberg, and B.I. Halperin, Rev. Mod. Phys. 49435 (1977); G. F. Mazenko, O. T. Valls, and F. C. Zhang, Phys. Rev. B31 4453 (1985); 325807 (1985) and A.J. Bray, Phys. Rev. Lett. 622841 (1989).

[7] J. L. Lebowitz, J. Marro and M. H. Kalos, Acta Metall. 30297 (1982)

[8] A. Cumming, P. Wiltzius and F. S. Bates, Phys. Rev. Lett. 65863 (1990); A. Cumming, P. Wiltzius, F. S. Bates and J. H. Rosendale, Phys. Rev. A 45885 (1992)

[9] M. Tokuyama and Y. Enomoto, Phys. Rev. Lett. 69312 (1992); Phys. Rev. E 471156 (1993)

[10] G. Coulon, B. Collin, D. Chatenay and Y. Gallot, J. Phys. II 3697 (1993)

[11] F. Despa and M. Apostol, Solid State Commun. 94153 (1995); J. Phys.\&Chem. Solids 571231 (1996); F. Despa and V. Topa, Z. Phys. D 3865 (1996); F. Despa, phys. stat. sol.(b), 19131 (1995)

[12] this result is obtained also for an interface kinetic limited growth, see J.A. Marqusee and J. Ross, J. Chem. Phys. 79373 (1983)

\section{Figure caption}

Fig. 1 - The growth of the surface area of a surface-melted cluster by the inter-cluster direct transfer mechanism (a) in comparison with the asymptotic regime of the Ostwald ripening process (b). The parameters employed in the present computation are: $v_{p-h}=210^{3} \mathrm{~s}^{-1}, E_{0}=0.6 \mathrm{eV}, E=0.82 \mathrm{eV}$, $k T=0.05 \mathrm{eV}$ and $\sigma=1 / 4 \AA^{-2}$. 
This figure "chicc4.jpg" is available in "jpg" format from: http://arxiv.org/ps/physics/0105073v1 